\title{
Off-Label Use of Phakic Intraocular Lens with a "Piggyback" Technique
}

\author{
Tadgh Schempf ${ }^{a}$ Hoon C. Jung ${ }^{b, c}$ \\ aNew York University School of Medicine, New York, NY, USA; bUniversity of Washington \\ Department of Ophthalmology, Seattle, WA, USA; 'Veterans Affairs Puget Sound \\ Health Care System, Seattle, WA, USA
}

\section{Keywords}

Cataract surgery · Phakic intraocular lens · High myopia · Piggyback technique $\cdot$ Refractive error

\begin{abstract}
Purpose: We report a case of a highly myopic pseudophakic patient who received off-label placement of a phakic intraocular lens (pIOL) via a "piggyback" technique, allowing the placement of an intraocular lens (IOL) in his fellow eye, resulting in improved visual acuity and emmetropia. Case Report: A 66-year-old, highly myopic, pseudophakic male with an IOL implant in his left eye was referred for second opinion for surgical options for his phakic right eye. Given the severe myopic status of both eyes, he received off-label placement of a posterior chamber pIOL with a piggyback technique for the pseudophakic left eye followed by standard cataract surgery and intraocular lens implantation in the right eye. For the left eye, uncorrected best visual acuity improved from 20/70 to 20/25. Conclusion: This case demonstrates the successful off-label use of a phakic IOL in a pseudophakic, highly myopic patient with a piggyback technique, resulting in improved visual acuity and ultimately allowing IOL placement in the fellow eye for emmetropia. This off-label use of plOL can offer ophthalmologists an alternative option for pseudophakic patents with severe refractive error.




\section{Introduction}

While outcomes of cataract surgery are excellent, the impetus to improve spectacle independence and emmetropia continues to be a measure of outcomes and satisfaction. Even with advances in formulae, biometric devices, and other adjunct technologies, there is room for additional precision. In the context of residual refractive error after cataract surgery, there are multiple options for correction including glasses, contact lenses, lens exchange surgery, and corneal refractive surgery [1-3]. Another option, however, is the placement of another intraocular lens (IOL) through the "piggyback" technique. This technique was first described by Gayton and Sanders [4] in 1993, who achieved a refractive power of $46.0 \mathrm{D}$ in a patient with microphthalmos. It involves the placement of both a primary and secondary IOL in the capsular bag [4]. This allows the patient to achieve greater refractive power than would be possible with one IOL alone. Since their advent, IOLs have been produced by many different manufacturers, and many different forms are used with the piggyback technique.

Phakic IOLs (pIOLs) are another type of lens that can be used in phakic patients with refractive error. Currently in the US, there exist two FDA-approved pIOLs, the iris-claw anterior chamber pIOL Verisyse (Abbott Laboratories Inc., Abbott Park, IL, USA) and posterior chamber pIOL Visian ICL (Staar Surgical, Monrovia, CA, USA) [5]. Phakic IOLs are most commonly used in young patients who have poor tolerance of glasses or contact lenses, poor tolerance of excimer laser surgery, and have stable refractive parameters [6]. The surgical technique of implantation depends on the type of pIOL: the Verisyse/Artisan iris-claw anterior chamber pIOL is fixated onto the anterior iris, and the Visian posterior chamber pIOL is injected similarly and tucked under the iris [6]. Clinical outcomes for pIOLs are impressive, exhibiting better best spectacle-corrected visual acuity (BCVA) and refractive stability than refractive surgery [7]. Visian posterior chamber pIOLs have been shown to have higher rates of emmetropia than Verisyse anterior chamber pIOLs [8]. Posterior chamber pIOLshave been shown to have good axial stability 6 months after surgery [9]. For operative planning, ultrasound biomicroscopy has utility in preoperative pIOL sizing [10]. There also exists another option for extracapsular "piggyback" IOL placement into the ciliary sulcus, using the Sulcoflex Pseudophakic Supplementary IOL (Rayner Intraocular Lenses Ltd., Worthing, UK) [11]. The use of these lenses has exhibited efficacy for correction of pseudophakic refractive error [11].

We present a case of a patient with bilateral severe myopia, right-eye cataract, and pseudophakic status in the left eye who requested evaluation for refractive procedure options. With a goal of offering increased spectacle independence, he received off-label placement of a pIOL with a piggyback technique for the left eye followed by standard cataract extraction and IOL implant for the right eye.

\section{Case Presentation}

A 66-year-old male was referred for evaluation for cataract surgery on his right eye. His ophthalmic history was notable for phacoemulsification and intraocular lens (IOL) implantation in his left eye (model type and power unknown) 13 years ago with large residual myopia. The patient was referred for a consultation for refractive surgery options.

His medical history was otherwise notable for hypertension, type 2 diabetes mellitus, hyperlipidemia, chronic obstructive pulmonary disease, prostate cancer (treated by total prostatectomy), and morbid obesity. His medications included glipizide, metformin, liraglutide, 
carvedilol, simvastatin, levothyroxine, and tiotropium. He reported no known allergies and denied tobacco or alcohol use.

On presentation, the patient reported poor vision at all distances, without any recent worsening of vision. BCVA was 20/70 and 20/200 in the right and left eyes, respectively, which corrected to $20 / 50$ and $20 / 70$ with pinhole. His near visual acuity was J3 bilaterally. His manifest refraction was $-14.00 \mathrm{D}+1.75 \times 075$ in his right eye and $-16.00 \mathrm{D}+1.00 \times 075$ in his left eye. Intraocular pressures (IOP) were 15 and 18 in his right and left eyes, respectively. Slit-lamp examination was notable for a 2+ nuclear sclerotic cataract in his right eye and a posterior chamber IOL with mild capsular fibrosis in his left eye (Fig. 1). Examination of the posterior segments was notable for pathologic myopia bilaterally, with signs of scleral thinning bilaterally (Fig. 2). Refractive analysis revealed compound myopic astigmatism bilaterally. Biometric analysis of both eyes showed notably long axial lengths $(30.5 \mathrm{~mm}$ right eye and $33.5 \mathrm{~mm}$ left eye) bilaterally (Fig. 3). Corneal tomography revealed normal corneal thickness and architecture bilaterally. After consideration of surgical options, the patient elected for the off-label placement of a phakic IOL in his left eye with a piggyback technique. Uncomplicated surgery was performed with the placement of a sulcus-located PIOL with a refractive power of $-16.00 \mathrm{D}$ and a diameter of $13.2 \mathrm{~mm}$ in the patient's left eye, using the technique described by Assetto et al. [12].

On postoperative day 1 , the patient returned for follow-up monitoring. He denied any new symptoms. The patient's uncorrected best visual acuity (UCVA) in his left eye was improved to $20 / 50$, with a pinhole visual acuity of $20 / 40$. His postoperative IOP was constantin his right eye, with a decrease in IOP to 16 in his left eye. Slit-lamp examination of his left eye was notable for a sutured wound on the temporal limbus, a pharmacologically dilated pupil, a deep and quiet anterior chamber, and visualization of the pIOL in correct position anterior to his prior IOL with a space between the two implants. His BCVA, IOP, and slit-lamp examination in his right eye remained stable from his preoperative evaluation. The patient returned 1 week later for ongoing monitoring and reported subjective improvementin his vision, with ongoing mild blurriness. In his left eye, UCVA was improved to $20 / 25$, and IOP was decreased to 13 . In his left eye, slit-lamp examination was notable for an intact sutured wound on the temporal limbus, a deep and quiet anterior chamber, and visualization of the pIOL in correct position anterior to his prior IOL with a space between the two implants (Fig. 4). His right-eye BCVA, IOP, and slit-lamp examination remained stable. The patient was then referred to his primary ophthalmologist for treatment of the nuclear sclerotic cataract in his right eye, with treatment aimed at emmetropia. He subsequently received a +4.0 D AMO SensAr Lens implant in the right eye with uncomplicated right eye surgery with 20/20 UCVA.

\section{Discussion}

This case illustrates the off-label "piggyback" use of a phakic IOL in a severely myopic pseudophakic patient to decrease spectacle dependence with emmetropic surgical refractive goals. This patient had marked improvement in his UCVA due to the placement of a pIOL anterior to a previously placed IOL, believed to be related to correcting refractive error at the principal optical planes. This ultimately allowed the placement of an IOL in the fellow eye and subsequent bilateral emmetropia.

As discussed previously, the typical FDA-approved use of pIOL technique involves the placement of an IOL in a phakic eye; however, the use of pIOLs in pseudophakic eyes with the piggyback technique remains an off-label use. There have been previous reports of pIOL 
Schempf and Jung: Off-Label Use of Phakic Intraocular Lens with a "Piggyback" Technique

placement via the piggyback technique in pseudophakic eyes. The first reported use of pIOLs in pseudophakic eyes was performed by Kojima et al. [13] in 2010, who showed in 8 pseudophakic eyes of 5 patients who underwent piggyback insertion of a pIOL to correct residual refractive error that pIOL placement in this situation was effective with predictable results. In a subsequent study by Hsuan et al. [14] of 6 pseudophakic patients with anisometropia who received pIOLs, anisometropia was decreased to asymptomatic levels, with a mean decrease in anisometropia of 3.15 D. Another case was described of an 80 -year-old female with pseudophakic ametropia who received a pIOL with marked improvement in visual acuity and elimination of anisometropia [15]. In 14 pediatric eyes with pseudophakic myopic anisometropic amblyopia, Eissa [16] described improved visual acuity and a decrease in manifest refraction spherical equivalent from -5.23 to $-0.30 \mathrm{D}$ with implantation of pIOLs. Eissa et al. [17] reproduced similar results in a larger cohort of 18 eyes of 18 pseudophakic, ametropic adults who received pIOLs.

The results from the patient presented in this report are similar to those previously reported, as he had improved vision and was able to pursue emmetropic IOL placement in the fellow eye. Notably, previous reports of this off-label use do not discuss fellow eye IOL placement as a major goal, representing a unique aspect of this case.

This off-label surgical technique offers ophthalmologists an alternative to standard methods of treating pseudophakic anisometropia. Firstly, as this patient was severely myopic, he preferred not to wear glasses with such a strong refractive power. Correction of this level of refraction would have been achievable optically with contact lenses, but not of interest for the patient. IOL exchange would have been exceedingly difficult for this patient, as slit-lamp examination showed marked capsular fibrosis involving the prior IOL. Further, manufacturing limits would have precluded the use of a lens with enough refractive power to achieve correction of $-15.00 \mathrm{D}$ of myopia. Corneal surgery, such as PRK or LASEK, would also not have been feasible for this patient, as his level of refractive error would have required removal of too much corneal tissue to maintain adequate minimal corneal thickness. As with all case reports, this represents a limited scope of evidence, and further investigation of this off-label technique is warranted to further evaluate its utility.

This case illustrates a novel off-label use of phakic IOL placement in the surgical management of pseudophakic myopia. Further study is warranted to characterize the possible benefits of this technique, as it could become an alternative option for ophthalmologists to offer patients with suboptimal outcomes after cataract surgery.

\section{Acknowledgements}

Work to present this case report was supported by the Research to Prevent Blindness (RPB).

\section{Statement of Ethics}

All personally identifiable information has been removed. The authors have no ethical conflicts. This case discusses the off-label surgical use of a product. 
Schempf and Jung: Off-Label Use of Phakic Intraocular Lens with a "Piggyback" Technique

\section{Disclosure Statement}

The authors have no conflicts of interest or proprietary interest in any of the topics or products presented in this paper.

\section{References}

1 Jin GJ, Crandall AS, Jones JJ. Intraocular lens exchange due to incorrect lens power. Ophthalmology. 2007 Mar;114(3):417-24.

2 Ayala MJ, Perez-Santonja JJ, Artola A, Claramonte P, Alio JL. Laser in situ keratomileusis to correct residual myopia after cataract surgery. J Refract Surg. 2001 Jan-Feb;17(1):12-6.

3 Zaldivar R, Oscherow S, Piezzi V. Bioptics in phakic and pseudophakic intraocular lens with the Nidek EC5000 excimer laser. J Refract Surg. 2002 May-Jun;18(3 Suppl):S336-9.

4 Gayton JL, Sanders VN. Implanting two posterior chamber intraocular lenses in a case of microphthalmos. J Cataract Refract Surg. 1993 Nov;19(6):776-7.

5 Huang D, Schallhorn SC, Sugar A, Farjo AA, Majmudar PA, Trattler WB, et al. Phakic intraocular lens implantation for the correction of myopia: a report by the American Academy of Ophthalmology. Ophthalmology. 2009 Nov;116(11):2244-58.

6 Güell JL, Morral M, Kook D, Kohnen T. Phakic intraocular lenses part 1: historical overview, current models, selection criteria, and surgical techniques. J Cataract Refract Surg. 2010 Nov;36(11):1976-93.

7 Barsam A, Allan BD. Excimer laser refractive surgery versus phakic intraocular lenses for the correction of moderate to high myopia. Cochrane Database Syst Rev. 2012 Jan;1:CD007679.

8 Karimian F, Baradaran-Rafii A, Hashemian SJ, Hashemloo A, Jafari ME, Yaseri M, et al. Comparison of three phakic intraocular lenses for correction of myopia. J Ophthalmic Vis Res. 2014 Oct-Dec;9(4):427-33.

9 Lin H, Yan P, Yu K, Luo L, Chen J, Lin Z, et al. Anterior segment variations after poster ior chamber phakic intraocular lens implantation in myopic eyes. J Cataract Refract Surg. 2013 May;39(5):730-8.

10 Dougherty PJ, Rivera RP, Schneider D, Lane SS, Brown D, Vukich J. Improving accuracy of phakic intraocular lens sizing using high-frequency ultrasound biomicroscopy. J Cataract Refract Surg. 2011 Jan;37(1):13-8.

11 Falzon K, Stewart OG. Correction of undesirable pseudophakic refractive error with the Sulcoflex intraocular lens. J Refract Surg. 2012;28(9):614-9.

12 Assetto V, Benedetti S, Pesando P. Collamer intraocular contact lens to correct high myopia. J Cataract Refract Surg. 1996 Jun;22(5):551-6.

13 Kojima T, Horai R, Hara S, Nakamura H, Nakamura T, Satoh Y, et al. Correction of residual refractive error in pseudophakic eyes with the use of a secondary piggyback toricImplantable Collamer Lens. J Refract Surg. 2010 Oct;26(10):766-9.

14 Hsuan JD, Caesar RH, Rosen PH, Rosen ES, Gore CL. Correction of pseudophakic anisometropia with the Staar Collamer implantable contact lens. J Cataract Refract Surg. 2002 Jan;28(1):44-9.

15 Chiou AG, Bovet J, de Courten C. Pseudophakic ametropia managed with a phakic posterior chamber intraocular lens. J Cataract Refract Surg. 2001 Sep;27(9):1516-8.

16 Eissa SA. Management of pseudophakic myopic anisometropic amblyopia with piggyback Visian® implantable collamer lens. Acta Ophthalmol. 2017 Mar;95(2):188-93.

17 Eissa SA, Khafagy MM, Sidky MK. Implantable Collamer Lens in the Management of Pseudophakic Ametropia. J Refract Surg. 2017 Aug;33(8):532-7. 


\section{Case Reports in Ophthalmology}

\begin{tabular}{l|l}
\hline Case Rep Ophthalmol 2018;9:465-472 \\
\hline DOI: 10.1159/000494712 & $\begin{array}{l}\text { ○ 2018 The Author(s). Published by S. Karger AG, Basel } \\
\text { www.karger.com/cop }\end{array}$ \\
\hline
\end{tabular}

Schempf and Jung: Off-Label Use of Phakic Intraocular Lens with a "Piggyback" Technique

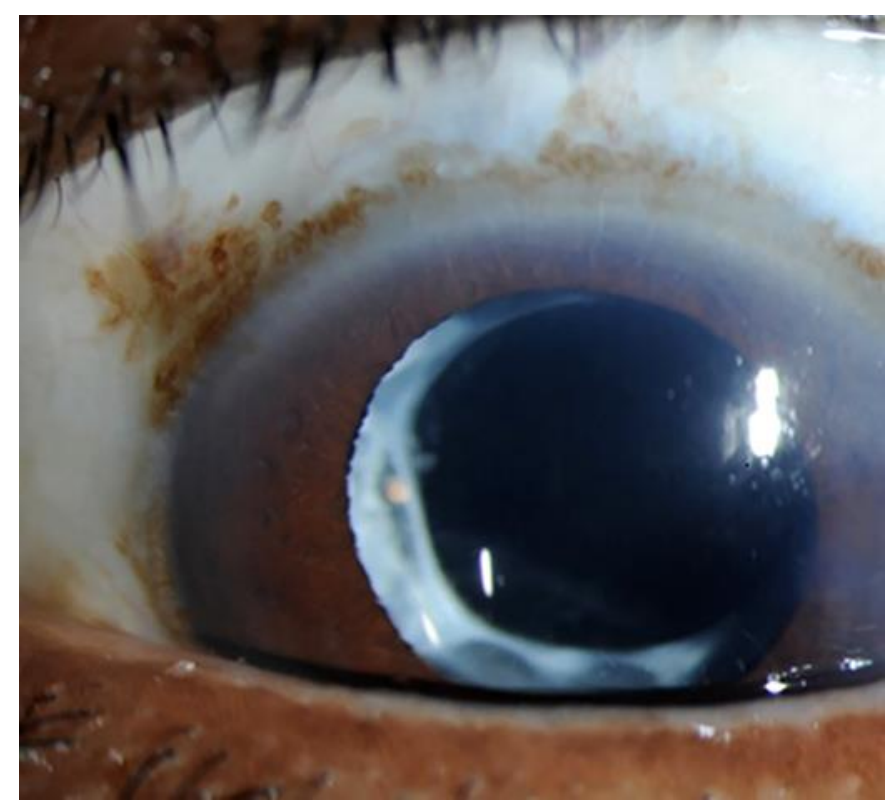

Fig. 1. Preoperative image of the left-eye anterior segment. IOL with capsular fibrosis is visualized.

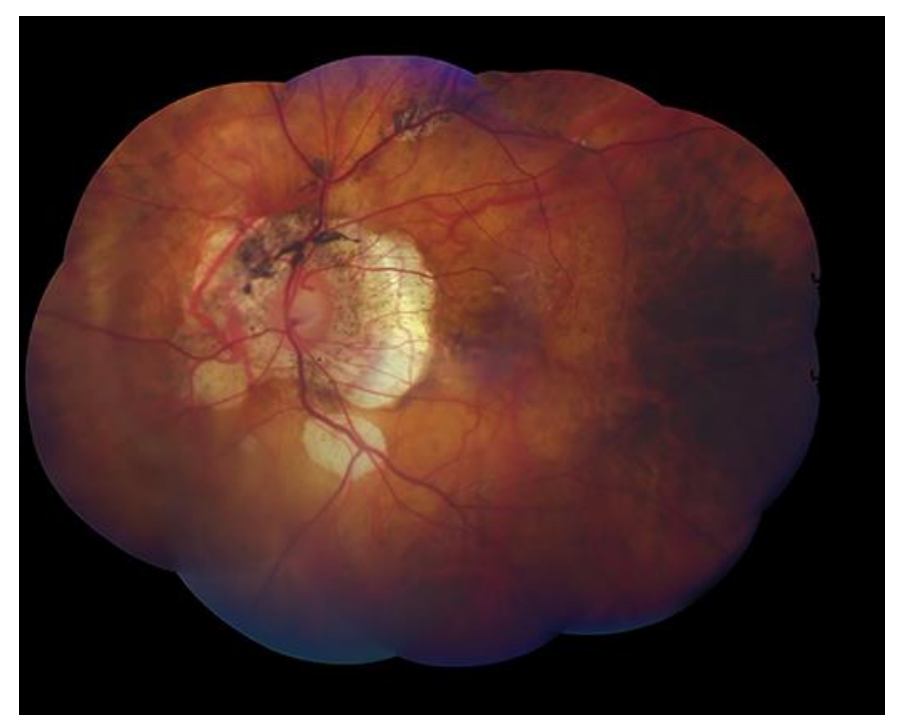

Fig. 2. Fundus photography of the left eye. Pathologic myopia with scleral thinning is visualized. 


\section{Case Reports in Ophthalmology}

\begin{tabular}{l|l}
\hline Case Rep Ophthalmol 2018;9:465-472 \\
\hline DOI: 10.1159/000494712 & $\begin{array}{l}\text { (c) 2018 The Author(s). Published by S. Karger AG, Basel } \\
\text { www.karger.com/cop }\end{array}$ \\
\hline
\end{tabular}

Schempf and Jung: Off-Label Use of Phakic Intraocular Lens with a "Piggyback" Technique

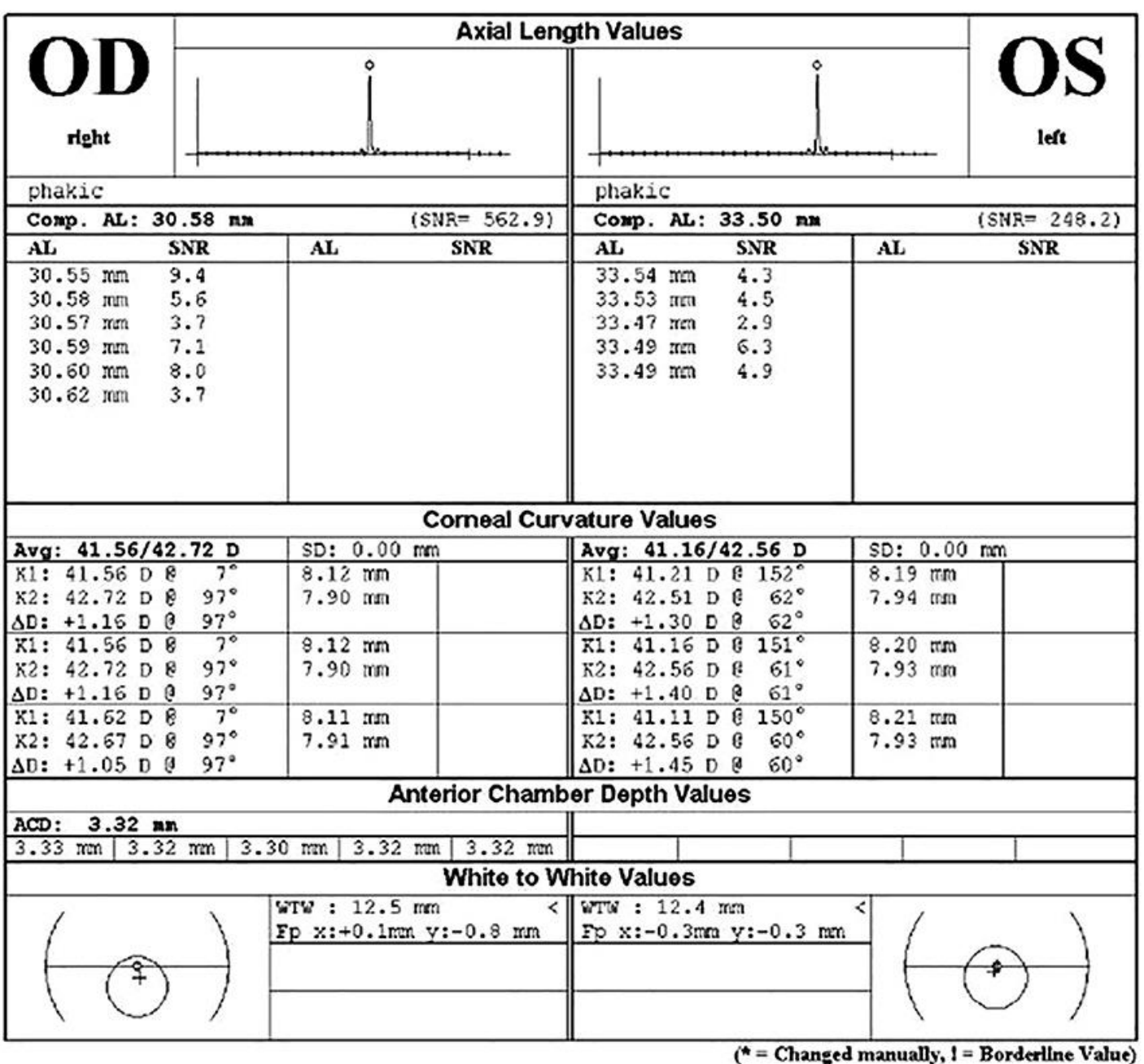

Fig. 3. Biometric analysis of both eyes. The evaluation is notable for pathologic myopia bilaterally. 


\section{Case Reports in Ophthalmology}

\begin{tabular}{l|l}
\hline Case Rep Ophthalmol 2018;9:465-472 \\
\hline DOI: 10.1159/000494712 & $\begin{array}{l}\text { @ 2018 The Author(s). Published by S. Karger AG, Basel } \\
\text { www.karger.com/cop }\end{array}$ \\
\hline
\end{tabular}

Schempf and Jung: Off-Label Use of Phakic Intraocular Lens with a "Piggyback" Technique
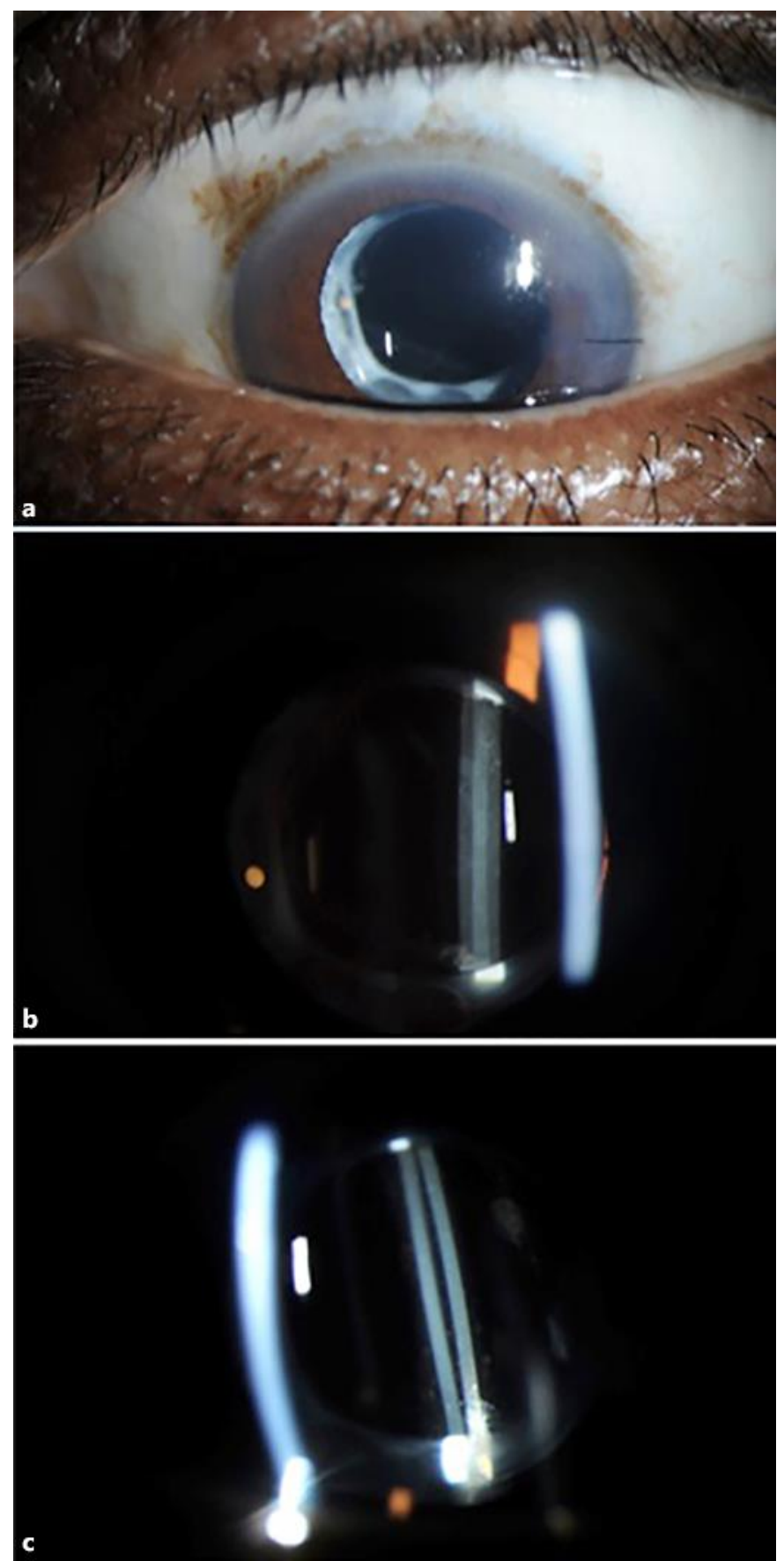

Fig. 4. Left-eye postoperative slit-lamp visualization of the anterior chamber and phakic IOL at 1 week. Lens power -16.0 D with a diameter of $13.2 \mathrm{~mm}$. a Anterior chamber with phakic IOL. b, c Slit-lamp visualization of phakic IOL and prior IOL implant. 